\title{
Design, Synthesis, and Biological Evaluation of Tetrahydroisoquinoline-Based Histone Deacetylase 8 Selective Inhibitors
}

Taha Y. Taha, ${ }^{\dagger}$ Shaimaa M. Aboukhatwa, ${ }^{\dagger, \dagger}$ Rachel C. Knopp, ${ }^{\dagger}$ Naohiko Ikegaki, ${ }^{\S}$ Hazem Abdelkarim, ${ }^{\dagger, \perp}$ Jayaprakash Neerasa, ${ }^{\dagger, \|}$ Yunlong Lu, ${ }^{\dagger}$ Raghupathi Neelarapu, ${ }^{\dagger, \nabla}$ Thomas W. Hanigan, ${ }^{\dagger}$ Gregory R. J. Thatcher, ${ }^{\dagger}$ and Pavel A. Petukhov* ${ }^{\dagger}+0$

${ }^{\dagger}$ Department of Medicinal Chemistry and Pharmacognosy, College of Pharmacy, University of Illinois at Chicago, Chicago, Illinois 60612, United States

${ }^{\ddagger}$ Department of Pharmaceutical Chemistry, Faculty of Pharmacy, Tanta University, Tanta 31527, Egypt

${ }^{\S}$ Department of Anatomy and Cell Biology, College of Medicine, University of Illinois at Chicago, Chicago, Illinois 60612, United States

Supporting Information

ABSTRACT: Histone deacetylase 8 (HDAC8) is a promising drug target for multiple therapeutic applications. Here, we describe the modeling, design, synthesis, and biological evaluation of a novel series of C1-substituted tetrahydroisoquinoline (TIQ)-based HDAC8 inhibitors. Minimization of entropic loss upon ligand binding and use of the unique HDAC8 "open" conformation of the binding site yielded a successful strategy for improvement of both
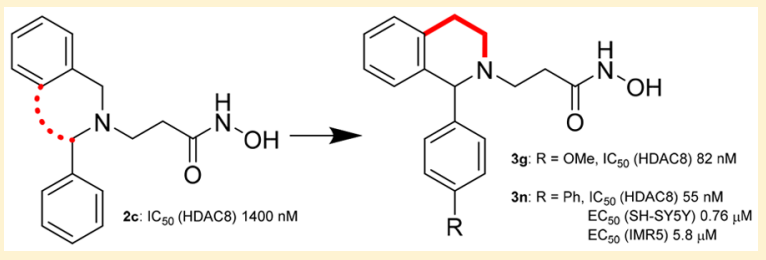
HDAC8 potency and selectivity. The TIQ-based $3 g$ and $3 \mathbf{n}$ exhibited the highest 82 and $55 \mathrm{nM}$ HDAC8 potency and 330- and 135-fold selectivity over HDAC1, respectively. Selectivity over other class I isoforms was comparable or better, whereas inhibition of HDAC6, a class II HDAC isoform, was below $50 \%$ at $10 \mu \mathrm{M}$. The cytotoxicity of $3 g$ and $3 \mathrm{n}$ was evaluated in neuroblastoma cell lines, and $3 \mathbf{n}$ displayed concentration-dependent cytotoxicity similar to or better than that of PCI-34051. The selectivity of $\mathbf{3 g}$ and $\mathbf{3 n}$ was confirmed in SH-SY5Y cells as both did not increase the acetylation of histone $\mathrm{H} 3$ and $\alpha$-tubulin. Discovery of the novel TIQ chemotype paves the way for the development of HDAC8 selective inhibitors for therapeutic applications.

KEYWORDS: Histone deacetylase, HDAC8, hydroxamate, inhibitor, tetrahydroisoquinoline

$\mathrm{H}$ istone deacetylases (HDACs), also known as lysine deacetylases, are enzymes that catalyze the removal of acetyl moieties from lysine $\varepsilon$-amino group residues in histones as well as nonhistone substrates. Deacetylation of histone substrates results in an overall change in the post-translational state of histones, known as the "histone code." ${ }^{1,2}$ The list of cellular events controlled by HDACs has grown beyond DNA replication, DNA repair, chromatin remodeling, and gene transcription to the world of noncoding microRNA, and it continues to expand. ${ }^{3,4}$ Normal regulation of these processes is compromised in a variety of disease states, and altered HDAC expression/function has been shown to be a hallmark of many cancers and neurodegenerative and inflammatory diseases. ${ }^{4-9}$ Because of the roles HDACs play in these diseases, HDAC inhibitors have emerged as therapeutics, with four FDAapproved HDAC inhibitors available to treat peripheral or cutaneous T-cell lymphoma and multiple myeloma.

Increasing evidence shows that HDAC isoforms carry out unique biological functions. ${ }^{10-12}$ Therefore, it has been proposed that desired therapeutic effects can be achieved by inhibiting a single or a subset of HDAC isoforms. ${ }^{13}$ This would also aid in minimizing clinical toxicities exhibited by the currently approved pan-HDAC inhibitors. ${ }^{14}$ HDAC8, a member of the class I HDAC family, has been investigated as a potential target in a variety of disease states, including neuroblastoma, ${ }^{15,16}$ acute myelogenous leukemia, ${ }^{17}$ colon cancer, ${ }^{18}$ schistosomiasis, ${ }^{19}$ and others. ${ }^{20}$ Recent analysis of tissues from breast cancer patients indicates that hypomethylation of HDAC8 promoter and HDAC8 overexpression correlate with disease progression and overall poor prognosis. ${ }^{21}$ To that end, HDAC 8 represents a promising therapeutic target with a somewhat limited set of potent isoform-selective inhibitors (Figure 1). ${ }^{16,20,22}$ Only aromatic hydroxamic acids are potent HDAC8 inhibitors, whereas aliphatic ones are less potent and inhibit other isoforms. $^{23-25}$ In this Letter, we present computer-aided molecular design, synthesis, and biological evaluation of novel amine-based series $\mathbf{1}$ and $\mathbf{2}$ and tetrahydroisoquinoline (TIQ)based HDAC8 selective inhibitors 3 .

Received: March 22, 2017

Accepted: July 26, 2017

Published: August 1, 2017 


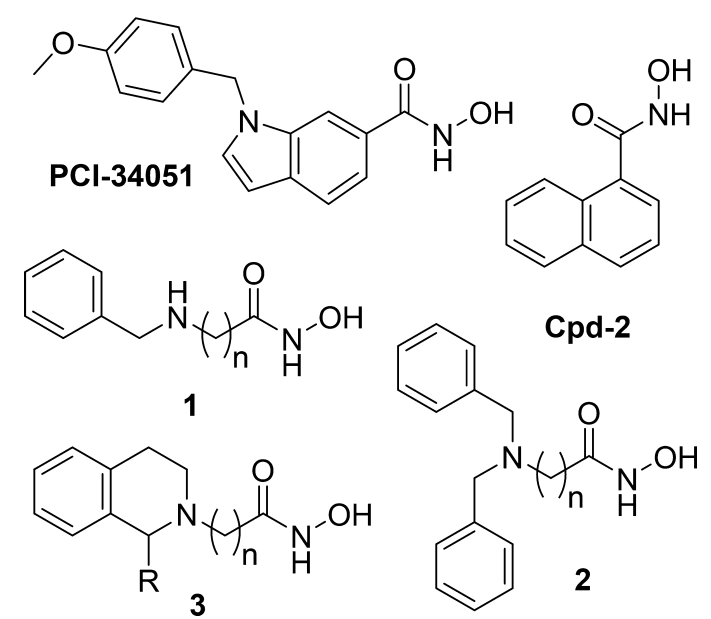

Figure 1. Structures of HDAC8 selective inhibitors PCI-34051 and Cpd-2 and general structures for secondary (1) and tertiary (2) aminebased and TIQ-based (3) HDAC inhibitors.

In our preliminary studies, we investigated the effect of linker length on potency of amine-based HDAC inhibitors. A library of novel secondary and tertiary amine-based compounds $1 \mathbf{1}-\mathbf{c}$ and $\mathbf{2 a}-\mathbf{c}$, respectively, was synthesized via alkylation of the corresponding primary amines and conversion of the methyl ester moiety to hydroxamic acid (Scheme 1). Screening of these compounds against class I HDAC isoforms (Table 1) showed that potency of secondary amines $\mathbf{1 a}-\mathbf{c}$ decreased for all class I isoforms as the length of the linker was shortened from six to two methylene groups. Potency of tertiary amines $\mathbf{2 a}-\mathbf{c}$ exhibited a similar trend only for HDAC1, 2, and 3, where it decreased from $1.1,2.7$, and $1.1 \mu \mathrm{M}$ for $2 \mathrm{a}(n=6)$ to $33,>100$, and $52 \mu \mathrm{M}$ for $2 \mathrm{c}$ $(n=2)$, respectively. However, shortening the linker of the tertiary amines led to a rather unexpected 2.9-fold improvement in potency for HDAC8 from $4.0 \mu \mathrm{M}$ for $2 \mathrm{a}$ to $1.4 \mu \mathrm{M}$ for $2 \mathrm{c}$. Changes in HDAC8 selectivity corresponding to shortening the linker were not appreciable for the secondary amines. The tertiary amines, however, experienced increased selectivity for HDAC8 when the linker was shortened with $2 \mathrm{c}$ being 24-, >71-, and 37-fold selective for HDAC8 over HDAC1, 2, and 3, respectively, compared to $2 \mathrm{a}$. Such substantial improvement in selectivity rendered it worth investigating further.

To guide further improvements in HDAC8 activity and selectivity, amines $1 \mathbf{a}-\mathbf{c}$ and $2 \mathbf{a}-\mathbf{c}$ were docked to the binding site of HDAC8 in the "closed" and "open" conformations ${ }^{26}$ and to the other class I HDAC isoforms. It was reported previously that binding of the "linkerless" ligands generally occurs in an open conformation of loop B (residues S30-K36), whereas ligands less bulky in the linker region bind to the conformation of HDAC8 with loop B closed. ${ }^{22,26,27}$ Analysis of the docking poses of the ligands in the "closed" conformation of HDAC 8 has shown that shortening the linker in secondary amines $1 \mathbf{a}-\mathbf{c}$ causes the benzyl moiety to bind deeper in the binding site. Overall, the secondary amines appear to be too small to cause pronounced conformational changes in the binding site, such as those found in the "open" conformation of HDAC8. Limited flexibility of the linker associated with a fewer number of methylene groups also limits the ability of the benzyl group to form more favorable hydrophobic interactions with aromatic residues lining the gorge region of the binding site. Altogether these observations may explain general loss of activity of secondary amines $\mathbf{1 a}-\mathbf{c}$ against class I HDAC isoforms. In the "closed" conformation of HDAC8, the two benzyl groups of tertiary amines $\mathbf{2 a}-\mathbf{c}$, and especially $\mathbf{2 c}$, share the rather narrow gorge of the binding site. The distance between the hydroxamate group in these ligands and the zinc ion in the binding site is longer, 2.6-2.9 $\AA$ for $\mathrm{Zn}^{2+} \ldots \mathrm{O}$ bonds, compared with $<2 \AA$ for the ligands crystallized with HDAC8, and the overall geometry of the zinc complex is distorted. ${ }^{28}$ Such pronounced deviations from the parameters typically observed in HDAC X-ray structures may result in substantially weaker binding. ${ }^{29}$ Since none of the crystal structures of HDAC1-3 have an "open" conformation, docking to these isoforms gave results very similar to those for the "closed" conformation of HDAC8, which is the likely reason for superior selectivity of $2 c$ for HDAC8. In the "open" conformation, however, $2 \mathrm{c}$ forms a more extended contact with the gorge region residues by occupying the additional lipophilic pocket (Figure 2A) between F152 and Y306 not available in the "closed" conformation, and the distance between the hydroxamate group and the zinc ion is similar to that observed in the X-ray structures. We hypothesized that an additional linker bridging the two benzyl groups in $2 \mathrm{c}$ as shown in Figure 2A may further improve potency of the resulting compounds by minimizing the entropic penalty paid by the benzyl groups upon binding.

To test this hypothesis, we designed and synthesized a series of TIQ-based compounds 3 and tested their activity against class I HDAC isoforms. To facilitate the synthesis, the length of the linker connecting the tertiary amine moiety and one of the phenyl rings (Figure 2A) was increased from one to two methylene groups. The general synthetic approach for the TIQbased compounds is shown in Scheme 2. For unsubstituted compounds, 1,2,3,4-tetrahydroisoquinoline was alkylated with the appropriate commercially available $\omega$-bromo esters with six or two methylene linkers. The resulting esters $\mathbf{6 a}-\mathbf{b}$ were then converted to the corresponding hydroxamic acids $3 \mathbf{a}-\mathbf{b}$. For $\mathbf{C} 1-$ substituted compounds, the starting commercially available acids $7 \mathbf{a}-\mathbf{j}$ were first converted to acyl chlorides and then coupled with phenethylamine resulting in amides $\mathbf{8 a}-\mathbf{j}$. Next, amides $\mathbf{8 a}-\mathbf{j}$ were subjected to Bischler-Napieralski cyclization and hydrogenation to give tetrahydroisoquinolines $\mathbf{9 a}-\mathbf{j}$. Intermediates $\mathbf{9 a}-\mathbf{j}$ were then alkylated with appropriate commercially available $\omega$-bromo esters with six, four, or two methylene linkers

Scheme 1. Synthesis of Amine-Based HDAC Inhibitors ${ }^{a}$

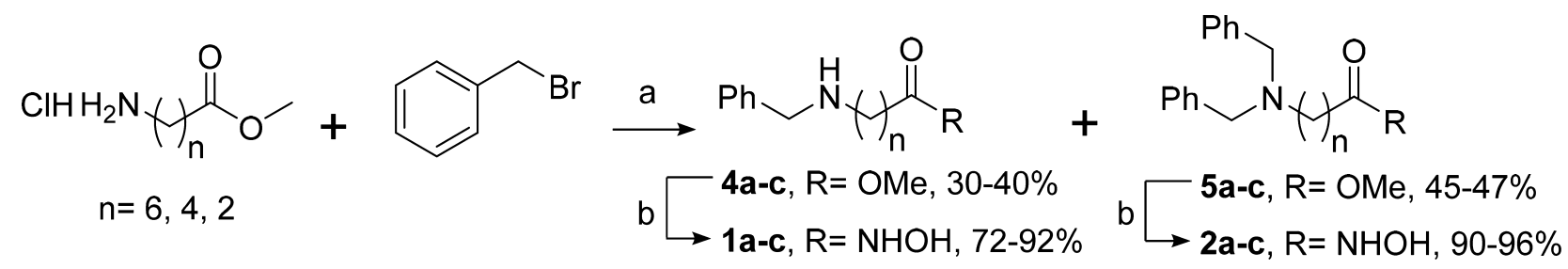

${ }^{a}$ Reagents and conditions: (a) $\mathrm{Et}_{3} \mathrm{~N}$ (1.5 equiv), $\mathrm{CH}_{3} \mathrm{CN}, \mathrm{rt}, 12 \mathrm{~h}$; (b) $\mathrm{NH}_{2} \mathrm{OH} \cdot \mathrm{HCl}$ (20 equiv), $\mathrm{KOH}\left(25\right.$ equiv), $\mathrm{MeOH}, 0{ }^{\circ} \mathrm{C}-\mathrm{rt}, 5 \mathrm{~h}$. 
Table 1. IC $_{50}$ Values of Amine-Based and TIQ-Based HDAC Inhibitors against Class I HDACs<smiles>[R]CN(CCCC(=O)NO)Cc1ccccc1</smiles>

1a-c, 2a-c

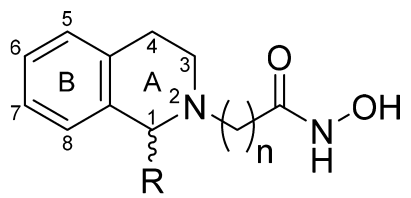

3a-n

\begin{tabular}{|c|c|c|c|c|c|c|c|}
\hline \multirow[b]{2}{*}{ compd } & \multirow[b]{2}{*}{$n$} & \multirow[b]{2}{*}{$\mathrm{R}$} & \multicolumn{4}{|c|}{$\mathrm{IC}_{50}(\mu \mathrm{M})^{a}$} & \multirow[b]{2}{*}{ HDAC6 (\% inhibition) $)^{b}$} \\
\hline & & & HDAC1 & HDAC2 & HDAC3 & HDAC8 & \\
\hline 1a & 6 & $\mathrm{H}$ & $0.34 \pm 0.0035$ & $3.1 \pm 0.79$ & $0.43 \pm 0.0038$ & $1.8 \pm 0.13$ & ND \\
\hline $1 b$ & 4 & $\mathrm{H}$ & $14 \pm 3.1$ & $58 \pm 0.96$ & $22 \pm 0.15$ & $10 \pm 3.2$ & ND \\
\hline 1c & 2 & $\mathrm{H}$ & $50 \pm 3.7$ & $>100$ & $72 \pm 4.2$ & $>100$ & $\mathrm{ND}$ \\
\hline $2 a$ & 6 & phenyl & $1.1 \pm 0.059$ & $2.7 \pm 0.67$ & $1.1 \pm 0.012$ & $4.0 \pm 0.37$ & $\mathrm{ND}$ \\
\hline $2 b$ & 4 & phenyl & $1.8 \pm 0.13$ & $5.4 \pm 0.23$ & $1.4 \pm 0.30$ & $1.9 \pm 0.20$ & $\mathrm{ND}$ \\
\hline $2 c$ & 2 & phenyl & $33 \pm 1.1$ & $>100$ & $52 \pm 3.0$ & $1.4 \pm 0.41$ & ND \\
\hline $3 a$ & 6 & $\mathrm{H}$ & $0.098 \pm 0.0038$ & $0.33 \pm 0.012$ & $0.097 \pm 0.0027$ & $1.9 \pm 0.17$ & $97 \pm 0.050$ \\
\hline $3 b$ & 2 & $\mathrm{H}$ & $57 \pm 3.2$ & $>100$ & $98 \pm 8.9$ & $19 \pm 2.4$ & $31 \pm 1.0$ \\
\hline $3 c$ & 4 & phenyl & $0.77 \pm 0.069$ & $2.7 \pm 0.12$ & $0.81 \pm 0.083$ & $0.044 \pm 0.026$ & $93 \pm 0.056$ \\
\hline $3 d$ & 2 & phenyl & $4.4 \pm 0.74$ & $40 \pm 2.0$ & $10 \pm 0.55$ & $0.95 \pm 0.012$ & $35 \pm 8.8$ \\
\hline $3 e$ & 1 & phenyl & $>100$ & $>100$ & $>100$ & $19 \pm 4.9$ & $17 \pm 5.1$ \\
\hline $3 f$ & 2 & 3-methoxyphenyl & $13 \pm 1.4$ & $69 \pm 11$ & $34 \pm 4.3$ & $0.11 \pm 0.026$ & $27 \pm 9.4$ \\
\hline $3 g$ & 2 & 4-methoxyphenyl & $27 \pm 3.7$ & $>100$ & $>100$ & $0.082 \pm 0.019$ & $21 \pm 0.040$ \\
\hline $3 \mathbf{h}$ & 2 & 4-ethoxyphenyl & $24 \pm 0.69$ & $99 \pm 4.9$ & $>100$ & $0.21 \pm 0.020$ & $5.7 \pm 4.5$ \\
\hline $3 \mathbf{i}$ & 2 & 3-(trifluoromethyl)phenyl & $37 \pm 3.1$ & $>100$ & $>100$ & $0.34 \pm 0.039$ & $17 \pm 6.1$ \\
\hline $3 \mathbf{j}$ & 2 & 4-(trifluoromethyl)phenyl & $18 \pm 3.6$ & $>100$ & $27 \pm 3.0$ & $0.19 \pm 0.033$ & $26 \pm 1.2$ \\
\hline $3 k$ & 2 & cyclohexyl & $>100$ & $>100$ & $75 \pm 4.9$ & $6.7 \pm 1.1$ & $32 \pm 0.080$ \\
\hline 31 & 2 & 4-(tert-butyl)phenyl & $18 \pm 2.7$ & $29 \pm 8.1$ & $29 \pm 13$ & $0.48 \pm 0.077$ & $30 \pm 2.0$ \\
\hline $3 m$ & 2 & naphthalene-2-yl & $2.4 \pm 0.23$ & $13 \pm 1.1$ & $3.4 \pm 0.21$ & $0.39 \pm 0.027$ & $74 \pm 0.25$ \\
\hline $3 n$ & 2 & 1,1'-biphenyl-4-yl & $7.3 \pm 0.48$ & $47 \pm 17$ & $38 \pm 2.2$ & $0.055 \pm 0.014$ & $39 \pm 1.2$ \\
\hline PCI-34051 & & & $3.8 \pm 2.7$ & $31 \pm 2.0$ & $7.1 \pm 0.38$ & $0.0066 \pm 0.0030$ & $37 \pm 4.1$ \\
\hline
\end{tabular}

${ }^{a} \mathrm{IC}_{50}$ values and ${ }^{b}$ percent inhibition of HDAC6 activity at $10 \mu \mathrm{M}$ concentration are expressed as mean \pm standard deviation of at least two independent experiments and rounded to two significant figures. ND: not determined.
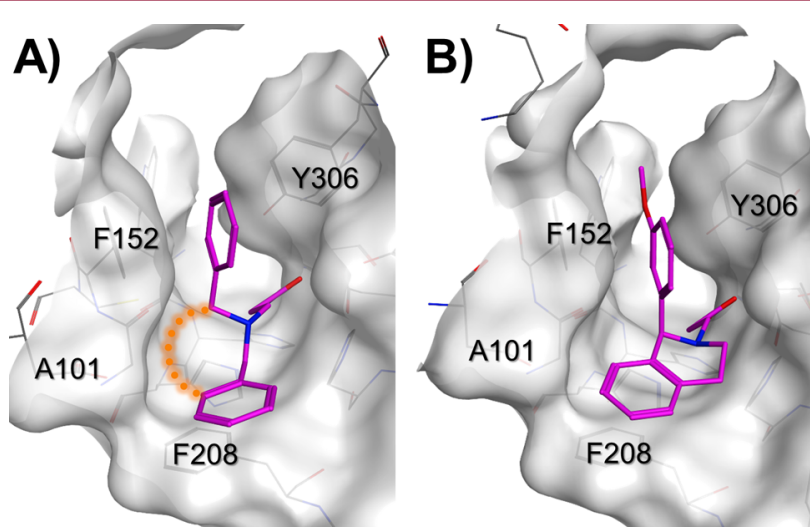

Figure 2. (A) Binding site in the "open" conformation of HDAC8 (PDB ID: $1 \mathrm{VKG}$ ) and the docking pose of $2 \mathrm{c}$. Possible rigidification is shown with the orange dotted line. (B) Same view of the binding site showing the docking pose of $\mathbf{3 f}$.

to give esters $10 a-1$, which were then converted to the corresponding racemic hydroxamic acids $3 \mathbf{c}-\mathbf{n}$. All the final compounds were tested for inhibition of class I HDAC isoforms. The $\mathrm{IC}_{50}$ inhibitory data for the known "linkerless" HDAC8 selective inhibitor PCI-34051 were generated in the same conditions in-house for comparison.

Unsubstituted TIQ-based compound 3a with a six-methylene linker had similar selectivity and up to 10 -fold higher potency for class I HDAC isoforms compared with the corresponding secondary amine 1a (Table 1). Shortening of the linker to two methylene groups in $\mathbf{3 b}$ resulted in loss of activity but somewhat improved selectivity for HDAC8. Modeling of the binding pose of the TIQ scaffold showed that the C1-substituent points into the HDAC8 extended pocket (Figure 2B), and varying this substituent would enable probing of the unique features of this pocket.

Substituents in the phenyl ring B at C5-C8, however, were expected to either point toward the solvent or to clash with the binding site (Figure 2B) and were deprioritized for this SAR study. To investigate the effect of C1 substitution in the TIQ scaffold, a series of compounds was synthesized, and the results are shown in Table 1. The TIQ scaffold was first substituted with a phenyl group, which resulted in $3 c-e$ closely resembling tertiary amines $2 a-c$, respectively. Compound $3 c$ with a fourmethylene linker had similar potency and selectivity for HDAC1, 2 , and 3, but had a 43-fold increase in potency and 18-, 61-, and 18-fold selectivity for HDAC8 over HDAC1, 2, and 3, respectively, compared with the corresponding tertiary amine $\mathbf{2 b}$. Shortening of the linker to two methylene groups resulted in compound 3d that exhibited 7.5-, 2.5-, 5.2-, and 1.5-fold improvement for HDAC1, 2, 3, and 8 potency, respectively. Its HDAC8 selectivity over HDAC1, 2, and 3 compared with the corresponding tertiary amine $2 \mathrm{c}$ decreased by 5.1 -, 1.7 -, and 3.5 fold, respectively. The observed slight improvement in potency of $3 \mathbf{d}$ for all class I isoforms might be due to HDAC1, 2, and 3 undergoing small conformational changes, similar to those in the "open" conformation of HDAC8. Further shortening of the linker to one methylene group in $3 \mathrm{e}$ resulted in a complete loss of 
Scheme 2. Synthesis of TIQ-Based HDAC Inhibitors ${ }^{a}$

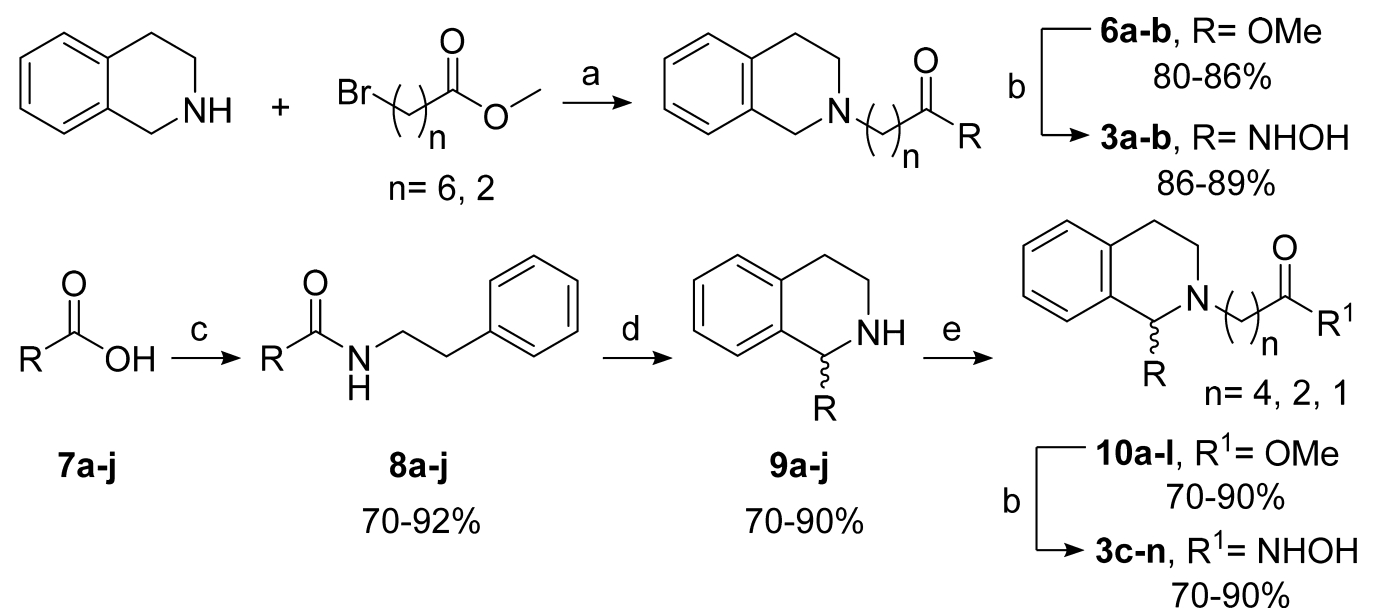

${ }^{a}$ Reagents and conditions: (a) methyl 3-bromopropanoate or methyl 7-bromoheptanoate (1.2 equiv), $\mathrm{K}_{2} \mathrm{CO}_{3}\left(2.0\right.$ equiv), $\mathrm{CH}_{3} \mathrm{CN}, \mathrm{rt}, 12 \mathrm{~h}$; (b) $\mathrm{NH}_{2} \mathrm{OH} \cdot \mathrm{HCl}$ (20 equiv), $\mathrm{KOH}\left(25\right.$ equiv), $\mathrm{MeOH}, 0{ }^{\circ} \mathrm{C}-\mathrm{rt}, 5 \mathrm{~h}$; (c) (i) $(\mathrm{COCl})_{2}\left(1.5\right.$ equiv), cat. $\mathrm{DMF}, \mathrm{CH}_{2} \mathrm{Cl}_{2}, 0^{\circ} \mathrm{C}, 3 \mathrm{~h}$; (ii) phenethylamine (1.0 equiv), $\mathrm{Et}_{3} \mathrm{~N}$ (1.5 equiv), $\mathrm{CH}_{2} \mathrm{Cl}_{2}, 0^{\circ} \mathrm{C}, 4 \mathrm{~h}$; (d) (i) $\mathrm{P}_{2} \mathrm{O}_{5}$ (2.5 equiv), $\mathrm{POCl}_{3}$ (3.0 equiv), xylene, reflux, $160{ }^{\circ} \mathrm{C}, 6 \mathrm{~h}$; (ii) $\mathrm{H}_{2} / \mathrm{PtO}_{2}$, ethanol, rt, $12 \mathrm{~h}$; (e) methyl 3-bromopropanoate, methyl 5-bromopentanoate, or methyl 7-bromoheptanoate (1.2 equiv), $\mathrm{K}_{2} \mathrm{CO}_{3}(2.0 \mathrm{equiv}), \mathrm{CH}_{3} \mathrm{CN}, \mathrm{rt}, 12 \mathrm{~h}$.

activity for HDAC1, 2, and 3 and showed only $19 \mu \mathrm{M}$ potency for HDAC8. Based on these findings, we decided to expand on the SAR studies of the C1-substituted TIQ-based compounds with two methylene groups between the hydroxamic acid zinc binding group and the nitrogen atom of the TIQ moiety.

To investigate the possible impact of electronic and steric effects on potency and selectivity of the compounds, a series of C1-substituted TIQ-based compounds were synthesized bearing electronically diverse substituents in the phenyl moiety. The meta- and para-methoxy substituted $\mathbf{3 f}$ and $\mathbf{3 g}$, respectively, had lower potency for HDAC1, 2, and 3 and 8.6- and 12-fold improved potency and selectivity for HDAC 8 compared with the unsubstituted $3 \mathrm{~d}$, respectively (Table 1 ). The slight 1.3 -fold increase in potency and selectivity for HDAC8 of the parasubstituted $3 \mathrm{~g}$ compared with the meta-substituted $3 \mathrm{f}$ suggests that the HDAC8 extended binding pocket is too narrow and prefers to accommodate linear $\mathrm{C} 1$ substituents (Figure 2B). The slight increase in size of the para-substituent in 4-ethoxyphenylTIQ $3 \mathrm{~h}$ resulted in nearly identical potency and selectivity compared to those of $\mathbf{3 g}$. Replacement of the electron donating methoxy substituent with an electron withdrawing trifluoromethyl moiety in $\mathbf{3} \mathbf{i}$ and $\mathbf{3} \mathbf{j}$ resulted in similar activity profiles to $3 \mathrm{f}$ and $\mathbf{3 g}$, respectively. The difference in potency for HDAC8 between meta and para substitution observed for $\mathbf{3 f}$ and $\mathbf{3 g}$, respectively, was also present in the trifluoromethyl substituted compounds, where $3 \mathbf{j}$ was 1.8 -fold more potent than $3 \mathbf{i}$. Compounds $3 \mathbf{i}$ and $3 \mathbf{j}$ exhibited a 3.1- and 2.3-fold decrease in potency for HDAC8 when compared with their meta- and parasubstituted methoxy analogues $3 \mathbf{f}$ and $\mathbf{3 g}$, respectively. The observed decrease in potency cannot be explained by electronic differences alone because the trifluoromethyl moiety is expected to increase potency via reducing the electron density on the face of the ring thereby decreasing repulsive component of the pi-pi interaction $^{30,31}$ between the phenyl moiety in the ligands and side chains of F152 and Y306 (Figure 2B). Overall, these results indicate that steric bulk of C1-substituent in the TIQ-based series plays a more important role in binding of the ligands to HDAC8.

To assess the possible effect of bulkier C1-substitunets on activity, compounds $3 \mathbf{k}-\mathbf{n}$ were synthesized and tested. Compound $3 \mathbf{k}$ containing a cyclohexyl substituent exhibited a pronounced loss of activity for HDAC1, 2, and 3 and only a 6.7 $\mu \mathrm{M}$ potency for HDAC8 compared with the phenyl-substituted 3d (Table 1). Interestingly, bulkier 4-(tert-butyl)phenyl C1substituent in 31 resulted in 4.1-fold lower potency for HDAC1, similar potency for HDAC2, 2.9-fold decrease in potency for HDAC3, and 2-fold improvement in potency for HDAC8 compared with $\mathbf{3 d}$. In contrast, the naphthyl substituted $3 \mathrm{~m}$ was 2- to 3-fold more potent for all the class $\mathrm{I}$ isoforms and maintained similar selectivity when compared to $3 \mathrm{~d}$. The drastic loss of HDAC 8 activity seen in $3 \mathbf{k}$ but not in $\mathbf{3 l}$ and $3 \mathbf{m}$ may, therefore, be explained by the general preference of the binding site for linear and flat aromatic substituents and inability to accommodate large nonflat groups, such as cyclohexyl, likely due to poor fit and loss of pi-pi interactions with the aromatic residues lining the gorge region of the binding site. To follow-up on these observations, we synthesized C1-biphenyl substituted compound $3 \mathrm{n}$ and found it to exhibit similar potency for HDAC2, 1.6- and 3.8-fold lower potency for HDAC1 and 3, respectively, and a 17 -fold higher potency for HDAC8 when compared with $3 \mathrm{~d}$. The improvement in potency for HDAC 8 but not for HDAC1, 2, or 3 of $3 \mathbf{n}$ compared to that of $3 \mathbf{k}, 31$, and $3 \mathbf{m}$ is consistent with the conformational changes observed only in the "open" conformation of HDAC8. These results suggest that not only aromatic "linkerless" but also aliphatic hydroxamic acidbased ligands are capable of causing conformational changes similar to those in the "open" conformation of HDAC8.

The TIQ-based inhibitors were docked to HDAC1-3 and 8 . Inhibitors demonstrating dual HDAC6 and 8 selectivity have been reported and include "linkerless" ${ }^{23,24}$ and substituted aliphatic hydroxamic acids, ${ }^{25}$ and hence, HDAC6 was also included in the docking studies and biological evaluation of the TIQ-based inhibitors. In all the isoforms, except the "open" conformation of HDAC 8 , the binding poses of $\mathbf{3 d}-\mathbf{n}$ were found to be similar to that of $\mathbf{2 c}$, i.e., suboptimal in geometry of binding to the zinc ion. Since some of the TIQ-based inhibitors show a low micromolar activity against $\operatorname{HDAC} 1,2$, and 3 , it is possible that these isoforms can also undergo small conformational changes, albeit probably not as extensive as those in HDAC8. HDAC6 activity assay showed that all the C1-substituted twomethylene linker TIQ-based compounds, except for $3 \mathrm{~m}$, 
exhibited less than $50 \%$ inhibition at $10 \mu \mathrm{M}$ concentration and were comparable to PCI-34051.

In silico analysis of the physicochemical properties of the amine-based and TIQ-based compounds demonstrated that these compounds are druglike and are likely to have excellent solubility and cell permeability (Supporting Information). The computed properties of these compounds are also in line with those active in the central nervous system (CNS). ${ }^{32}$ Considering outstanding selectivity of our TIQ-based inhibitors even for very homologous class I HDACs, there is a high probability that offtarget activity against other metalloproteases is minimal. Somewhat similar TIQ-containing hydroxamic acids, although without a basic nitrogen, showed only weak inhibition of aminopeptidase $\mathrm{N}^{33}$

Given their high potency and selectivity for HDAC8, compounds $3 \mathrm{~g}$ and $3 \mathrm{n}$ were evaluated in neuroblastoma cell lines. Compound 3n exhibited significant concentration- and time-dependent cytotoxicity similar to or better than PCI-34051 in all the cell lines (Figures 3A, Supporting Information).

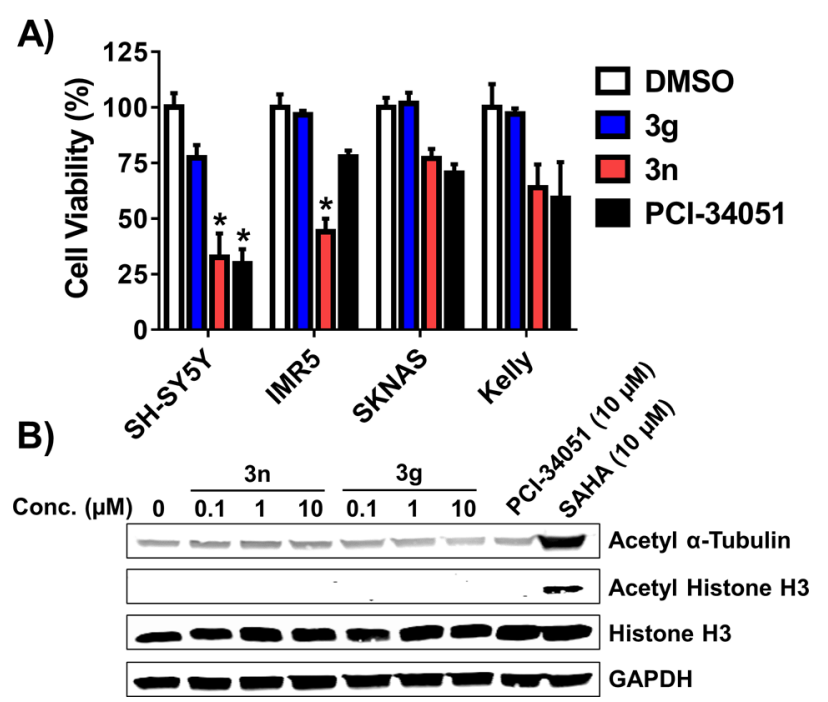

Figure 3. Cytotoxicity and HDAC8 selectivity of $\mathbf{3 g}$ and $3 \mathbf{n}$ in neuroblastoma cells. (A) Cells were treated with $10 \mu \mathrm{M} \mathrm{3g}$ or $3 \mathrm{n}$ for 48 $\mathrm{h}$, and cell proliferation was assessed by MTS assay. (B) Cells were treated with indicated concentrations for $24 \mathrm{~h}$ followed by Western blot analysis of acetylation of histone $\mathrm{H} 3$ and $\alpha$-tubulin. Cell viability values represent mean \pm standard deviation of at least three independent experiments. *Statistically significant difference compared with DMSO control (Student's $t$ test, $p<0.001$ ). Blot is representative of three independent experiments.

Compound 3n was most potent in SH-SY5Y $\left(\mathrm{EC}_{50}=0.76 \mu \mathrm{M}\right)$ and IMR5 $\left(\mathrm{EC}_{50}=5.8 \mu \mathrm{M}\right)$ cell lines after $48 \mathrm{~h}$ treatment. Compound $3 \mathrm{~g}$ showed pronounced activity only at $10 \mu \mathrm{M},>72 \mathrm{~h}$ treatment, and in SH-SY5Y cells (Supporting Information). The differences in cytotoxicity of $\mathbf{3 g}$ and $\mathbf{3 n}$ (Figure 3A) despite the similar HDAC inhibitory profile might be due to target engagement differences in cells compared with recombinant enzyme assays as well as off-target activity. The solubility or cell permeability are unlikely to play a role based on their precited properties. The selectivity of $\mathbf{3 n}$ and $\mathbf{3} \mathbf{g}$ for HDAC8 among other class I and II HDACs was confirmed in SH-SY5Y cells. Both compounds did not affect the acetylation of $\alpha$-tubulin or histone H3 similar to PCI-34051 compared with nonselective SAHA (Figure 3B).
In summary, the modeling, design, synthesis, SAR studies, and biological evaluation of the amine-based and C1-substituted TIQ-based compounds led to the discovery of the novel series of highly potent and selective HDAC8 inhibitors. Rigidification of the substituents connected to the tertiary amino group to minimize entropic loss upon binding presents an example of successful strategy for improvement of both HDAC8 potency and selectivity. The difference in propensity of class I HDAC isoforms to undergo conformational changes such as those in the "open" conformation of HDAC8 appears to account for the unique potency and selectivity of the C1-substituted TIQ-based compounds. The SAR studies suggest that binding of linear and aromatic substituents in the additional lipophilic pocket in the "open" conformation of HDAC8 is preferred. We envision that such rational approach to inducing the conformational changes in HDAC8 may be a valuable addition to the drug design toolbox for HDACs and other targets. Future studies will focus on improvement of HDAC8 potency and selectivity by varying the substitution at the $\mathrm{C} 1$ position as well as exploring the effects of substituting the TIQ scaffold at other positions.

\section{ASSOCIATED CONTENT}

S Supporting Information

The Supporting Information is available free of charge on the ACS Publications website at DOI: 10.1021/acsmedchemlett.7b00126.

Chemical, biological, and molecular modeling methods and experimental data (PDF)

\section{AUTHOR INFORMATION}

Corresponding Author

*E-mail: pap4@uic.edu. Phone: 312-996-4174.

ORCID

Taha Y. Taha: 0000-0002-7344-7490

Thomas W. Hanigan: 0000-0003-0752-4707

Gregory R. J. Thatcher: 0000-0002-7757-1739

Pavel A. Petukhov: 0000-0003-1554-242X

\section{Present Addresses}

${ }^{\perp}$ Department of Biochemistry and Molecular Genetics, College of Medicine, University of Illinois at Chicago, Chicago, Illinois 60607, United States.

"Department of Research \& Development, DongBang Future Tech \& Life Co. Ltd., Seoul, South Korea.

${ }^{\nabla}$ Department of Chemistry and Biochemistry, University of Delaware, Newark, Delaware 19716, United States.

\section{Author Contributions}

The manuscript was written through contributions of all authors. All authors have approved the final version of the manuscript.

Notes

The authors declare no competing financial interest.

\section{ACKNOWLEDGMENTS}

This study was funded by the National Cancer Institute/NIH grants R21 CA183627, R01 CA131970, and R01 HL130760, the Alzheimer's Drug Discovery Foundation grant ADDF \#20101103, and UICentre for Drug Discovery.

\section{ABBREVIATIONS}

HDAC, histone deacetylase; HDACs, histone deacetylases; TIQ, tetrahydroisoquinoline; SAR, structure-activity relationship; CNS, central nervous system 


\section{REFERENCES}

(1) Jenuwein, T.; Allis, C. D. Translating the histone code. Science 2001, 293, 1074-80.

(2) Gardner, K. E.; Allis, C. D.; Strahl, B. D. Operating on chromatin, a colorful language where context matters. J. Mol. Biol. 2011, 409, 36-46.

(3) Delcuve, G. P.; Khan, D. H.; Davie, J. R. Roles of histone deacetylases in epigenetic regulation: emerging paradigms from studies with inhibitors. Clin. Epigenet. 2012, 4, 5.

(4) Xu, W. S.; Parmigiani, R. B.; Marks, P. A. Histone deacetylase inhibitors: molecular mechanisms of action. Oncogene 2007, 26, 554152.

(5) Falkenberg, K. J.; Johnstone, R. W. Histone deacetylases and their inhibitors in cancer, neurological diseases and immune disorders. Nat. Rev. Drug Discovery 2014, 13, 673-691.

(6) Johnstone, R. W. Histone-deacetylase inhibitors: novel drugs for the treatment of cancer. Nat. Rev. Drug Discovery 2002, 1, 287-299.

(7) Marks, P.; Rifkind, R. A.; Richon, V. M.; Breslow, R.; Miller, T.; Kelly, W. K. Histone deacetylases and cancer: causes and therapies. Nat. Rev. Cancer 2001, 1, 194-202.

(8) Minucci, S.; Pelicci, P. G. Histone deacetylase inhibitors and the promise of epigenetic (and more) treatments for cancer. Nat. Rev. Cancer 2006, 6, 38-51.

(9) Li, Z.; Zhu, W. G. Targeting histone deacetylases for cancer therapy: from molecular mechanisms to clinical implications. Int. J. Biol. Sci. 2014, 10, 757-70.

(10) Dovey, O. M.; Foster, C. T.; Cowley, S. M. Histone deacetylase 1 (HDAC1), but not HDAC2, controls embryonic stem cell differentiation. Proc. Natl. Acad. Sci. U. S. A. 2010, 107, 8242-8247.

(11) Deardorff, M. A.; Bando, M.; Nakato, R.; Watrin, E.; Itoh, T.; Minamino, M.; Saitoh, K.; Komata, M.; Katou, Y.; Clark, D.; Cole, K. E.; De Baere, E.; Decroos, C.; Di Donato, N.; Ernst, S.; Francey, L. J.; Gyftodimou, Y.; Hirashima, K.; Hullings, M.; Ishikawa, Y.; Jaulin, C.; Kaur, M.; Kiyono, T.; Lombardi, P. M.; Magnaghi-Jaulin, L.; Mortier, G. R.; Nozaki, N.; Petersen, M. B.; Seimiya, H.; Siu, V. M.; Suzuki, Y.; Takagaki, K.; Wilde, J. J.; Willems, P. J.; Prigent, C.; Gillessen-Kaesbach, G.; Christianson, D. W.; Kaiser, F. J.; Jackson, L. G.; Hirota, T.; Krantz, I. D.; Shirahige, K. HDAC8 mutations in Cornelia de Lange syndrome affect the cohesin acetylation cycle. Nature 2012, 489, 313-7.

(12) Bhaskara, S.; Knutson, S. K.; Jiang, G.; Chandrasekharan, M. B.; Wilson, A. J.; Zheng, S.; Yenamandra, A.; Locke, K.; Yuan, J.-1.; BonineSummers, A. R.; Wells, C. E.; Kaiser, J. F.; Washington, M. K.; Zhao, Z.; Wagner, F. F.; Sun, Z.-W.; Xia, F.; Holson, E. B.; Khabele, D.; Hiebert, S. $\mathrm{W}$. Hdac3 is essential for the maintenance of chromatin structure and genome stability. Cancer Cell 2010, 18, 436-447.

(13) Balasubramanian, S.; Verner, E.; Buggy, J. J. Isoform-specific histone deacetylase inhibitors: the next step? Cancer Lett. 2009, 280, 211-21.

(14) Lane, A. A.; Chabner, B. A. Histone deacetylase inhibitors in cancer therapy. J. Clin. Oncol. 2009, 27, 5459-68.

(15) Oehme, I.; Deubzer, H. E.; Wegener, D.; Pickert, D.; Linke, J. P.; Hero, B.; Kopp-Schneider, A.; Westermann, F.; Ulrich, S. M.; von Deimling, A.; Fischer, M.; Witt, O. Histone deacetylase 8 in neuroblastoma tumorigenesis. Clin. Cancer Res. 2009, 15, 91-9.

(16) Rettig, I.; Koeneke, E.; Trippel, F.; Mueller, W. C.; Burhenne, J.; Kopp-Schneider, A.; Fabian, J.; Schober, A.; Fernekorn, U.; von Deimling, A.; Deubzer, H. E.; Milde, T.; Witt, O.; Oehme, I. Selective inhibition of HDAC8 decreases neuroblastoma growth in vitro and in vivo and enhances retinoic acid-mediated differentiation. Cell Death Dis. 2015, 6, e1657.

(17) Durst, K. L.; Lutterbach, B.; Kummalue, T.; Friedman, A. D.; Hiebert, S. W. The inv(16) fusion protein associates with corepressors via a smooth muscle myosin heavy-chain domain. Mol. Cell. Biol. 2003, 23, 607-19.

(18) Kang, Y.; Nian, H.; Rajendran, P.; Kim, E.; Dashwood, W. M.; Pinto, J. T.; Boardman, L. A.; Thibodeau, S. N.; Limburg, P. J.; Lohr, C. V.; Bisson, W. H.; Williams, D. E.; Ho, E.; Dashwood, R. H. HDAC8 and STAT3 repress BMF gene activity in colon cancer cells. Cell Death Dis. 2014, 5, e1476.
(19) Heimburg, T.; Chakrabarti, A.; Lancelot, J.; Marek, M.; Melesina, J.; Hauser, A. T.; Shaik, T. B.; Duclaud, S.; Robaa, D.; Erdmann, F.; Schmidt, M.; Romier, C.; Pierce, R. J.; Jung, M.; Sippl, W. StructureBased Design and Synthesis of Novel Inhibitors Targeting HDAC8 from Schistosoma mansoni for the Treatment of Schistosomiasis. J. Med. Chem. 2016, 59, 2423-35.

(20) Balasubramanian, S.; Ramos, J.; Luo, W.; Sirisawad, M.; Verner, E.; Buggy, J. J. A novel histone deacetylase 8 (HDAC8)-specific inhibitor PCI-34051 induces apoptosis in T-cell lymphomas. Leukemia 2008, 22, 1026-34.

(21) Hsieh, C. L.; Ma, H. P.; Su, C. M.; Chang, Y. J.; Hung, W. Y.; Ho, Y. S.; Huang, W. J.; Lin, R. K. Alterations in histone deacetylase 8 lead to cell migration and poor prognosis in breast cancer. Life Sci. 2016, 151, $7-14$.

(22) Krennhrubec, K.; Marshall, B. L.; Hedglin, M.; Verdin, E.; Ulrich, S. M. Design and evaluation of 'Linkerless' hydroxamic acids as selective HDAC8 inhibitors. Bioorg. Med. Chem. Lett. 2007, 17, 2874-8.

(23) Olson, D. E.; Wagner, F. F.; Kaya, T.; Gale, J. P.; Aidoud, N.; Davoine, E. L.; Lazzaro, F.; Weiwer, M.; Zhang, Y. L.; Holson, E. B. Discovery of the first histone deacetylase 6/8 dual inhibitors. J. Med. Chem. 2013, 56, 4816-20.

(24) Rodrigues, D. A.; Ferreira-Silva, G. A.; Ferreira, A. C.; Fernandes, R. A.; Kwee, J. K.; Sant'Anna, C. M.; Ionta, M.; Fraga, C. A. Design, Synthesis, and Pharmacological Evaluation of Novel N-Acylhydrazone Derivatives as Potent Histone Deacetylase 6/8 Dual Inhibitors. J. Med. Chem. 2016, 59, 655-70.

(25) Negmeldin, A. T.; Padige, G.; Bieliauskas, A. V.; Pflum, M. K. Structural Requirements of HDAC Inhibitors: SAHA Analogues Modified at the C2 Position Display HDAC6/8 Selectivity. ACS Med. Chem. Lett. 2017, 8, 281-286.

(26) Brunsteiner, M.; Petukhov, P. A. Insights from comprehensive multiple receptor docking to HDAC8. J. Mol. Model. 2012, 18, 39273939.

(27) Tabackman, A. A.; Frankson, R.; Marsan, E. S.; Perry, K.; Cole, K. E. Structure of 'linkerless' hydroxamic acid inhibitor-HDAC8 complex confirms the formation of an isoform-specific subpocket. J. Struct. Biol. 2016, 195, 373-8.

(28) Dowling, D. P.; Gantt, S. L.; Gattis, S. G.; Fierke, C. A.; Christianson, D. W. Structural studies of human histone deacetylase 8 and its site-specific variants complexed with substrate and inhibitors. Biochemistry 2008, 47, 13554-63.

(29) Harding, M. M. The geometry of metal-ligand interactions relevant to proteins. II. Angles at the metal atom, additional weak metaldonor interactions. Acta Crystallogr., Sect. D: Biol. Crystallogr. 2000, 56, $857-67$.

(30) Rashkin, M. J.; Waters, M. L. Unexpected substituent effects in offset pi-pi stacked interactions in water. J. Am. Chem. Soc. 2002, 124, 1860-1.

(31) Sinnokrot, M. O.; Sherrill, C. D. Substituent effects in pi-pi interactions: sandwich and T-shaped configurations. J. Am. Chem. Soc. 2004, 126, 7690-7.

(32) Ghose, A. K.; Herbertz, T.; Hudkins, R. L.; Dorsey, B. D.; Mallamo, J. P. Knowledge-Based, Central Nervous System (CNS) Lead Selection and Lead Optimization for CNS Drug Discovery. ACS Chem. Neurosci. 2012, 3, 50-68.

(33) Flipo, M.; Beghyn, T.; Charton, J.; Leroux, V. A.; Deprez, B. P.; Deprez-Poulain, R. F. A library of novel hydroxamic acids targeting the metallo-protease family: design, parallel synthesis and screening. Bioorg. Med. Chem. 2007, 15, 63-76. 